\title{
Biological factors in eating and its disorders
}

\author{
NEIL E. ROWLAND \\ University of Florida, Gainesuille, Florida
}

\begin{abstract}
This short review is intended to illustrate some of the approaches that psychobiologists use to analyze processes underlying food intake in animals. The pivotal role that the concept of homeostasis continues to occupy is discussed first. There follows a review of some of the metabolic changes in the hypothalamus as a function of altered nutritional status. It is suggested that these changes can determine feeding in novel circumstances, but that they may have less control over learned feeding responses. The concept of separate appetites for the major macronutrients is evaluated, and the notion is introduced that the two sides of the hypothalamus may be metabolically different. Some of the neurotransmitters that have been implicated in feeding are mentioned, and the way in which such basic information may contribute to the treatment of overeating and obesity is illustrated.
\end{abstract}

It has often been argued that the adult body weight of an individual is maintained relatively constant (homeostasis), and such constancy in the milieu intérieur (after Claude Bernard) implies a regulatory process. Traditional models of active regulation in biological systems include some form of negative feedback process that is invariably responding to a change of state (reactive mode). However, this kind of model falls short of describing even simple physiological control systems (Houk, 1988). It is therefore unlikely that behavior can be modeled in terms of such devices. Instead, terms such as "adaptive control" and "predictive regulation" have been proposed to describe (albeit in "black box" form) ways in which systems operations are modified, respectively, by learned and anticipated (e.g., nycthemeral) factors (see, e.g., Davis, 1980; Rowland, 1990; Weingarten, 1990).

Over the past decades, psychobiologists have eschewed this type of analysis for reductionist hypothesis testing, usually of the single-factor negative feedback variety. In the examples that follow, the pervasiveness of this type of thinking should be evident. However, the results need to be interfaced with "real world" behavioral strategies such as food procurement and ingestion (Collier, 1989).

\section{METABOLIC FUEL HYPOTHESES OF EATING}

In an influential synthesis of the literature, Friedman and Stricker (1976) argued that feeding behavior was related to the current availability of oxidizable fuels (viz., glucose, fatty acids, ketones). Nicolaidis uses the term ischymetry for the measurement of current fuel utilization (Gk. ischys = "power"). These ideas embody the principle of the interchangeability of metabolic fuels, especially glucose and fats. Nicolaidis and Even (1989) have

Correspondence should be addressed to Neil Rowland, Department of Psychology, University of Florida, Gainesville, FL 32611-2065. reported that single cells in the hypothalamus that are responsive to application of glucose (glucoreceptors) also respond to other classes of fuels (ketones). Kasser, Harris, and Martin (1985) reported changes in hypothalamic fuel metabolism that are regionally selective and that parallel liver metabolism (Figure 1). These data and other evidence demonstrate that metabolic fuel status affects cellular processes in specific hypothalamic areas that often have been involved reciprocally in feeding.

\section{Antimetabolites and Feeding}

Antimetabolites are chemicals that interfere with normal fuel utilization; they also increase food intake and have figured prominently in metabolic models of feeding. Glucose antimetabolites include 2-deoxy-D-glucose (2DG) and engage " glucoprivic feeding" (Epstein, Nicolaidis, \& Miselis, 1975) by means of a cerebral mechanism. Injection of large doses of insulin causes hypoglycemia and probably engages the same mechanism (Rowland \& Stricker, 1979). Fat antimetabolites include mercaptoacetate (MA) and evoke feeding by a neural mechanism of peripheral origin (Ritter \& Taylor, 1989). As can be seen in Figure 2, simultaneous administration of glucose and fat antimetabolites produces a very large feeding response in rats (Ritter \& Taylor, 1989) and also mimics starvation insofar as both treatments cause hamsters to become anestrous (Schneider \& Wade, 1989). Indirectly, these effects of combined blockade show that glucose and fat are alternate fuels, because blockade of either alone produces much smaller effects.

The liver is a potential site for the transduction of both glucose and fat-related metabolism, and information from the liver may be integrated in the brainstem and/or hypothalamus. In addition, selected parts of the hypothalamus have liver-like metabolism (Figure 1).

\section{Infusions of Metabolic Fuels}

The natural counterpart of the antimetabolite paradigm is the provision of excess energy and the bypassing of the mouth. In general, parenteral injections or infusions of 


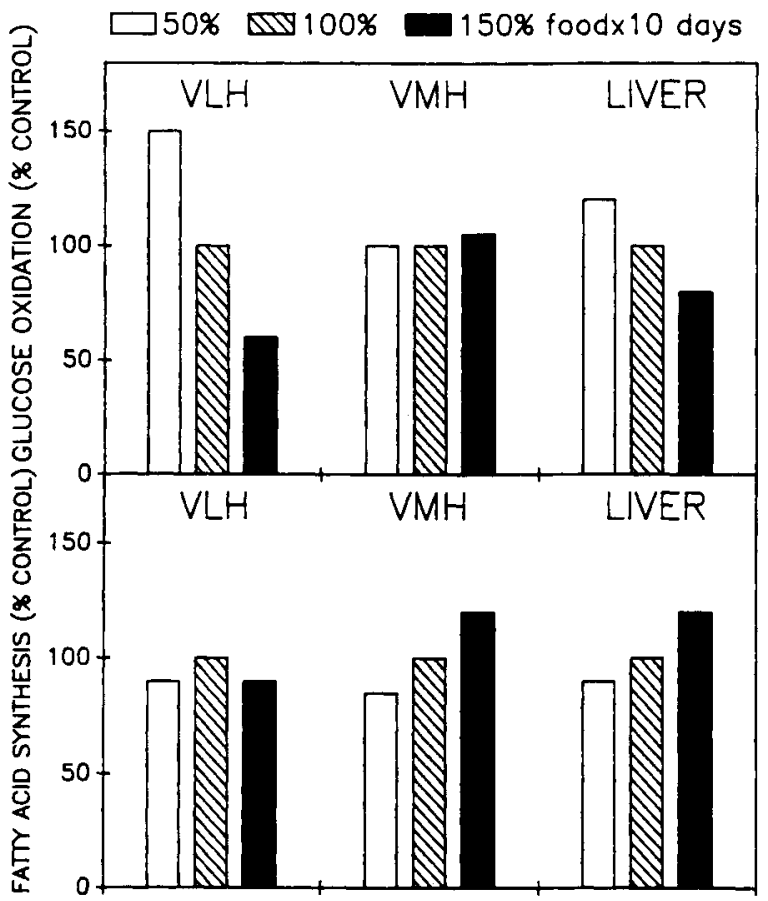

Figure 1. The effects of tube-feeding rats for $\mathbf{1 0}$ days with reduced, normal, or excess amounts of food relative to free-feeding baseline, on glucose oxidation and fatty acid synthesis in dissected regions of the hypothalamus (VMH = ventromedial; VLH = ventrolateral) and the liver. The data are expressed as percentages of the data in the normal intake group. Glucose oxidation changes in VLH and in the liver, and fatty acid oxidation changes in VMH and in the liver. Redrawn from Kasser, Harris, and Martin (1985).

either single metabolic fuels or mixtures decrease voluntary food intake. The suppressions are greater for infusates that contain many types of fuel (Nicolaidis \& Rowland, 1976) but are less than quantitative (i.e., 1 kcal infused lowers intake by $<1 \mathrm{kcal})$. However, when fuels are administered either in the presence of exogenous insulin, or as discrete i.v. "meals," the suppression of feeding is nearly quantitative.

As an extension, we showed that feeding induced acutely by insulin was inhibited by infusions of either glucose or fructose (Stricker, Rowland, Saller, \& Friedman, 1977). During those studies, we noted that glucose became less effective as animals were tested repeatedly, so insulin plus glucose infusion was usually the first test. In view of the recent interest in learned factors and feeding (Weingarten, 1990; see also the papers by Birch, 1991, and Sclafani, 1991 , in this issue), I recently returned to this paradigm to examine the effects of glucose and fructose on insulininduced feeding in rats that received two consecutive insulin tests.

On the 1st test day, all rats received insulin followed by a saline, glucose, or fructose infusion, i.v. On the 2 nd test day, the infusion conditions were changed. Of relevance here are the rats that received insulin plus saline and then insulin plus glucose in Tests 1 and 2, respectively, compared with those treated with insulin plus glu- cose in the first test. As expected, the latter group (Glul, Figure 3) ate very little in Test 1 . In contrast, rats that received insulin plus saline on the 1st day showed only $50 \%$ suppression of feeding by glucose in the second test (Glu2). Also, in contrast to our original work, and for reasons that are not clear, fructose was nearly ineffective on either day (Frul and Fru2). Conditioned responses to insulin occur quite quickly (see Woods \& Kulkosky, 1976), but these data indicate that a single metabolic emergency can drastically modulate the effects of parenteral fuels. Aside from the implications for the (lack of) effects of fuels on everyday (learned) feeding, this paradigm may provide a way to assess some of the components of the "adaptive control" device.

If the effects on intake are less than clear, do infusions have reinforcing effects? Tordoff, Tepper, and Friedman

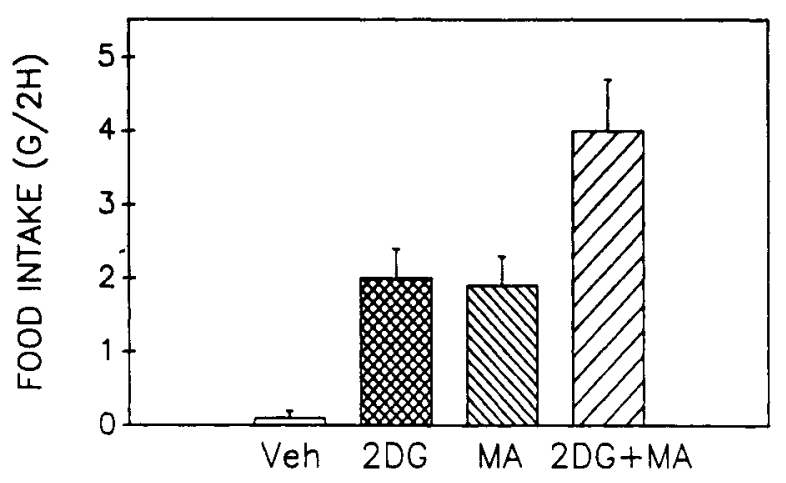

Figure 2. Mean ( $\pm S E$ ) 2-h intake of chow by rats injected with the glucose antimetabolite 2DG (100 mg/kg, s.c.), the fat antimetabolite mercaptoacetate (MA: $\mathbf{4 0 0} \mu \mathrm{mol} / \mathbf{k g}$, i.p.), or the combination. Redrawn from Ritter and Taylor (1989).

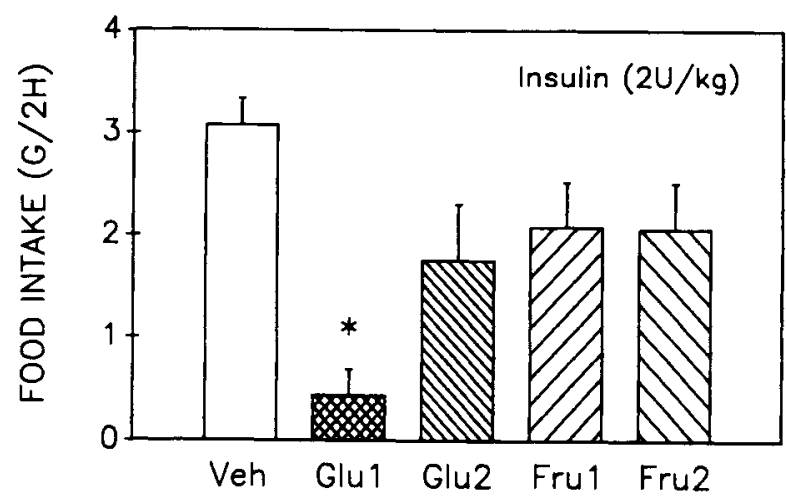

Figure 3. Mean $( \pm S E)$ 2-h intake of chow by rats injected with insulin (2U/kg, s.c.) followed by a $2-\mathrm{h}$ intravenous infusion of saline (Veh, $3 \mathrm{ml} / \mathrm{h}$ ), glucose (Glu, $1.2 \mathrm{M}, 7.2 \mathrm{mmol} / 2 \mathrm{~h}$ ), or fructose (Fru, 1.2 M). The Veh group is an average of animals receiving insulin either on Test Day 1 only or on both Days 1 and 2 (the intakes on these two tests did not differ). The Glul group are rats that received insulin and glucose infusion on Day 1 . The Glu2 group are rats that received insulin-vehicle on Day 1 and insulin-glucose on Day 2. Likewise for Frul and Fru2. ${ }^{*} p<.01$ versus insulin-vehicle. (Test Days 1 and 2 were 2-3 days apart.) $N /$ group $=6-8$. Rowland, 1990, unpublished data. 
(1987) showed that taste preferences can be formed on the basis of fuel oxidation when the fuels are administered per os (taste to utilizable calorie conditioning). However, Nicolaidis and Rowland (1977) attempted to train rats to press a lever to obtain i.v. nutrients. Although the rats did show some indications of regulation, their daily i.v. intakes were $<50 \%$ of normal oral intakes, and all of the rats lost large amounts of weight.

Systemic cues alone thus have a fairly weak influence on behavior. Factors including taste and learned incentive values seem to either predominate over, or at least act with, systemic factors in determining the size of a meal and the duration of satiety therefrom. On neuroanatomical grounds, it is likely that brainstem nuclei, including the nucleus tractus solitarius, could serve such an integrative role, and units in this nucleus alter their taste responsiveness as a result of physiological state and/or learning (Scott, 1990). Much work remains to be done on the specific mechanisms by means of which internal (metabolic) and external (smell, taste) signals are integrated within the context of concepts such as adaptive control, mentioned earlier. It is likely, for example, that systemic factors play a major role during learning about new foods (including during ontogeny; see Birch, 1991), but that learning then becomes part of an "expectancy" and the metabolic signals will subsequently be attended to only if some unforeseen emergency arises.

\section{NEUROCHEMICAL CORRELATES OF INGESTION}

Ultimately, the flow of information in the brain is mediated by neurotransmitters and modulators. Major advances have been made in both direct measurement of and indirect pharmacological assessment of transmitters involved in eating behavior. However, as with the physiological work, studies of neurochemical systems have focused on approaches based on synaptic homeostasis. I will briefly discuss a few such systems, as well as their relation to metabolism (Table 1).

\section{Orexigenic Agents}

There are relatively few putative transmitters that, when given centrally to sated animals, will increase food intake. The best-documented are norepinephrine (NE), neuropeptides Y (NPY) and YY, galanin, and kappa-opioid agonists (Table 1). NE has a maximal orexigenic effect in the paraventricular nucleus of the medial hypothalamus (PVN) and acts at postsynaptic alpha-2 type receptors (Leibowitz, 1988). In rats, these receptors increase in number in the early night, apparently driven by the nycthemeral rhythm in plasma glucocorticoid levels; maximal NE feeding occurs at this time. Further, Leibowitz has argued that rats self-selecting their diet from pure macronutrient sources take more carbohydrate (CHO) early in the night, and because $\mathrm{NE}$ injection preferentially increases $\mathrm{CHO}$ intake, NE may be involved in CHO-specific appetite.

This idea that separate neurochemical systems may underlie selection of the different macronutrients is not
Table 1

Agents That Increase and Decrease Food Intake

\begin{tabular}{lc}
\hline \multicolumn{1}{c}{ Orexigenic } & \multicolumn{1}{c}{ Anorexigenic } \\
\hline Glucose antimetabolites & Peripheral \\
2,5-anhydro-D-mannitol & Metabolic fueis \\
Insulin (high dose) & Cholecystokinin \\
& $\begin{array}{l}\text { Glucagon } \\
\text { Thyrotropin-releasing hormone } \\
\text { Insulin (low dose) }\end{array}$ \\
& Central \\
Norepinephrine (PVN) & Norepinephrine \\
Neuropeptide Y and YY & Dopamine-amphetamines \\
Galanin & Serotonin-fenfluramine \\
Opioids (kappa agonist) & Corticotropin-releasing factor \\
Benzodiazepines & Oxytocin \\
\hline
\end{tabular}

Note-This is a partial list. For more complete accounts, see Bray, Fisher, and York (1990), Morley (1987), and Woods, Taborsky, and Porte (1986).

readily compatible with the metabolic hypotheses mentioned above, which emphasize the interchangeability of fuels, particularly fats and CHO (see Drewnowski, 1991, for further comment). Also, although there is good evidence that caloric intake is in some sense regulated, there is little evidence that either fat or carbohydrate intakes are individually regulated. An example of this from a study by Ashley (1985) is shown in Figure 4. When a nutritionally complete food (energy) is made available at increasing cost (fixed ratio barpress), rats conserved intake up to high ratios (see Collier, 1989, for further review of work schedules and meal patterns). When rats were instead allowed to self-select among three pure macronutrients, each of which was available from a separate lever so that the cost of one nutrient could be manipulated independently of the cost of the other two, it was found that responding for $\mathrm{CHO}$ essentially ceased when CHO was more costly than fat. In contrast, they worked to maintain a certain minimum protein intake when this

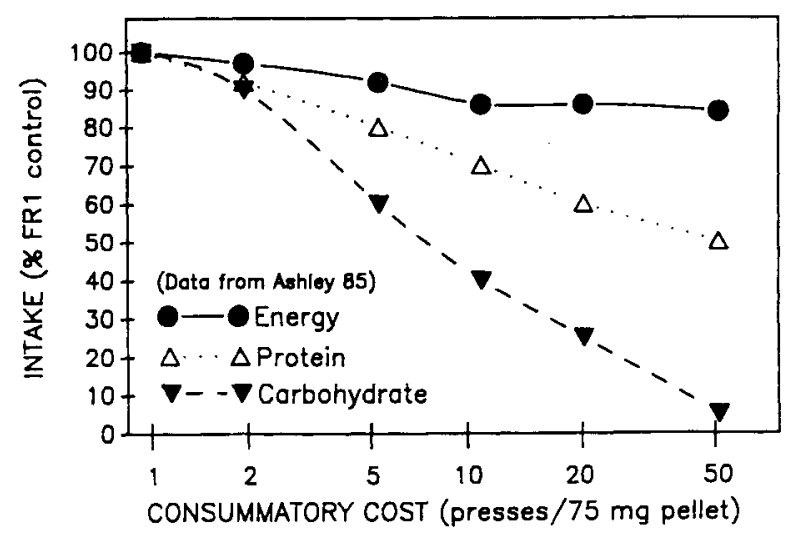

Figure 4. Mean daily food intake as a function of cost, expressed relative to the intake with fixed ratio (FR)1, of rats working either for nutritionally complete pellets (Energy), or in a 3-lever selfselection station, in which either protein or carbohydrate was increased in cost while the other two macronutrients remained "cheap." Note that rats do not work hard to maintain carbohydrate intake (but instead eat fat, not shown). Redrawn from Ashley (1985). 
was the costly commodity. This is strong evidence against a specific appetite for $\mathrm{CHO}$, at least as a biochemical commodity. Rats metabolically and behaviorally readily substitute a less costly option.

The PVN is also the "hot spot" for NPY-induced feeding and chronic cerebral administration of NPY leads to obesity (Stanley, Anderson, Grayson, \& Leibowitz, 1989). We have shown that NPY-induced feeding is reduced by i.v. infusions of glucose, but not fructose (Rowland, 1988), suggesting that metabolic fuel availability acts downstream of NPY in the control of feeding.

The relationship between metabolism, neurotransmitters, and feeding has been studied after injection of glucoprivic agents (Bellin \& Ritter, 1981; Rowland, Bellush, \& Carlton, 1985). Hypothalamic NE turnover is increased by insulin and 2DG, and, although this does not prove that NE causes feeding in this paradigm, it can be used as a marker of brain action. In earlier studies (Rowland et al., 1985), we found that 2DG-induced NE turnover was similar in both the lateral and the medial hypothalamus of rats; these regions traditionally have been reciprocally related for ingestion (see Figure 1), which argues against a causal role of NE release in feeding.

Recently, we have examined whether NE turnover is the same on both sides of the brain (since the vagal input to the brainstem is laterally asymmetrical), and also whether it is increased by fat as well as glucose antimetabolites. We found that 2DG statistically increased NE turnover (assessed by decline in NE levels after inhibition of synthesis) in the left but not in the right hypothalamus (Figure 5). We have found this greater effect on the left as opposed to the right hypothalamus in several other studies, although it is not always statistically significant. In addition, the fat antimetabolite alone had no effect, suggesting a peripheral site of action, but in combination with

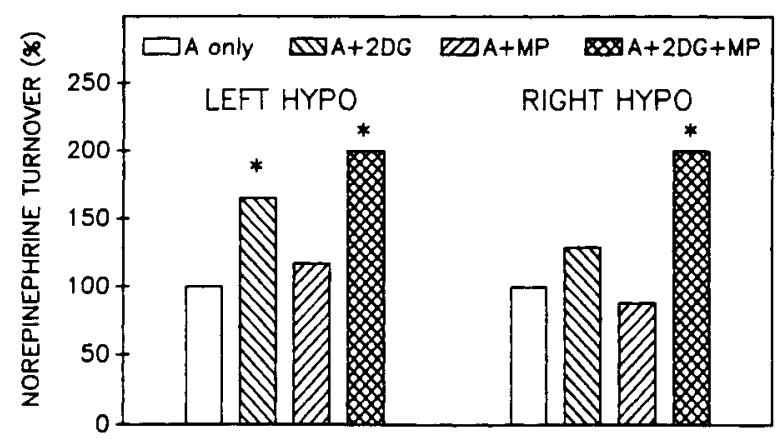

Figure 5. Norepinephrine turnover in the left and right hypothalamus of rats treated with $2 D G(200 \mathrm{mg} / \mathrm{kg}$, i.p.), the fat antimetabolite methylpalmoxirate (10 mg/kg orally, $1 \mathrm{~h}$ beforehand), or the combination. All rats received $\alpha$-methyl-p-tyrosine (A: $250 \mathrm{mg} / \mathrm{kg}$, s.c., an inhibitor of NE synthesis) and were killed $2 \mathrm{~h}$ later for determination of NE levels by HPLC-ED. The turnover rate of the group receiving A only (and calculated relative to no-drug true controls, data not shown) was set at $100 \%$, and the antimetabolite groups are expressed relative to this value. ${ }^{*} p<.05$ increased NE turnover. Note that the 2DG effect is significant only on the left. N/group $=6$. Rowland, 1990, unpublished data.
2DG, the fat antimetabolite further increased NE turnover on both sides (Figure 5), an effect that parallels the increased feeding (Figure 2). These studies suggest that the two sides of the hypothalamus may be in part specialized to respond to different metabolic signals. In view of the lateral/medial differences in other metabolic work (Figure 1), this issue also needs to be revisited with the combined fat and glucose antimetabolite paradigm. These results are preliminary, and I emphasize that the generality of the data and their implication for the organization of eating behavior remain to be established. Within the broader context of this paper, it is also worth noting that these results are typical of those obtained in emergency or "reactive" paradigms.

\section{Anorexigenic Agents}

The list of agents that decrease feeding in Table 1 is by no means complete, and is longer than the list of orexigenic agents. This suggests that the brain has multiple inhibitory mechanisms relevant to feeding. Among the endogenous agents studied most are insulin (in the cerebrospinal fluid and brain) and cholecystokinin (CCK: released into the blood during meals). Insulin and CCK occur in both the brain and its periphery, and relatively little is known about their release from the brain systems. The evidence rests on findings that central injections or infusions of insulin or CCK reduce food intake (see Morley, 1987; Woods, Taborsky, \& Porte, 1986, for reviews). Other candidate anorexigenic transmitters include corticotropin-releasing factor (CRF) and serotonin (5HT). Both of these are anorectic after central injection, and, in the case of genetically obese (but not lean) rats, continuous infusion of CRF reduces food intake and weight gain (Bray, Fisler, \& York, 1990). This has led to the notion that CRF has an orchestrational role with respect to feeding.

Wurtman and Fernstrom (reviewed in Fernstrom, 1983) were the first to show that alterations in CHO metabolism could have effects on brain 5HT synthesis, and an argument has been made that increased 5HT inhibits $\mathrm{CHO}$ appetite (Wurtman \& Wurtman, 1988).

\section{IMPLICATIONS FOR EATING DISORDERS}

How should we define an eating disorder and whether or not it should be treated? Should we use a "medical model" based on health risks, or a "behavioral/ecological model" that emphasizes shorter term gains for the animal and its progeny? Any biopsychologist has to opt for the latter, although we are frequently asked for solutions to deviations from the former. The short-term risks of anorexia and bulimia nervosa are more serious than those of overeating and subsequent obesity and often need prompt professional intervention.

There are two conceptually different ways in which psychobiologists can approach eating disorders. One is that the physiology of feeding was or is defective (at some level) and might be corrected. The second is that there 
is nothing "wrong" with the specific feeding systems, but that some aspects of (say) "adaptive control" have become unusually influential over behavior. In this case, intervention in feeding circuits might alleviate the symptom (e.g., overeating) without treating the causes of the symptom.

If we can agree on what symptoms should be treated, we need to treat both the causes and the symptoms. There are no convincing animal models of anorexia/bulimia (Smith, 1989), but hyperphagia and overweight may be stimulated in rats by varied foods, fat diets, stress, and other factors (Bray et al., 1990; Sclafani, 1989). These types of obesity can be alleviated by chronic treatment with clinically effective anorectic agents such as dexfenfluramine (DFEN) (Carlton \& Rowland, 1989; Rowland \& Carlton, 1988; Souquet \& Rowland, 1990). The most likely mechanism by means of which DFEN causes anorexia involves increases in 5HT availability in central synapses (Rowland \& Carlton, 1988). Interestingly, in two of the few published studies of neurochemical changes at the initiation of feeding (Holmes, Smythe, \& Storlien, 1989; Schwartz, McClane, Hernandez, \& Hoebel, 1989), increased 5HT activity in the hypothalamus was correlated with onset of feeding and/or cephalic phase insulin release. Thus, agents such as DFEN may amplify the normal neurochemical signals that occur during the earliest phases of a meal and so hasten satiety. It should be noted that the animals in the two studies mentioned above were conditioned to expect food at a particular time, so these neurochemical responses are not unique to first exposure to the stimuli. DFEN is anorectic under comparable test conditions (Souquet \& Rowland, 1989).

One of the challenges for future studies is to go beyond single-system or single-transmitter models of eating disorders, as well as to assess specificity. (For example, the release of dopamine in striatum is increased by anticipation of a meal; see Blackburn, Phillips, Jakubovic, \& Fibiger, 1989; Holmes, Smythe, \& Storlien, 1989.) This may open the way to a better understanding of whom to treat and how, including combinations of drug regimens and behavioral modification.

\section{REFERENCES}

ASHLEY, D. V. M. (1985). Factors affecting the selection of protein and carbohydrate from a dietary choice. Nutrition Research, 5, 555-571.

BELLIN, S. I., \& RITTER, S. (1981). Disparate effects of infused nutrients on delayed glucoprivic feeding and hypothalamic norepinephrine turnover. Journal of Neuroscience, 1, 1347-1353.

BIRCH, L. L. (1991). Obesity and eating disorders: A developmental perspective. Bulletin of the Psychonomic Society, 29, 265-272.

Blackburn, J. R., Phillips, A. G., Jakubovic, A., \& Fibiger, H. C. (1989). Dopamine and preparatory behavior: II. A neurochemical analysis. Behavioral Neuroscience, 103, 15-23.

Bray, G. A., Fisler, J., \& York, D. A. (1990). Neuroendocrine control of the development of obesity: Understanding gained from studies of experimental animal models. Frontiers in Neuroendocrinology, 11, 128-181.

Carlton, J., \& Rowland, N. E. (1989). Long term actions of $d$-fenfluramine in two rat models of obesity: I. Sustained reductions in body weight and adiposity without depletion of brain serotonin. International Journal of Obesity, 13, 825-847.

Collier, G. (1989). The economics of hunger, thirst, satiety and regulation. Annals of the New York Academy of Sciences, 575, 136-154.

DAvis, J. D. (1980). Homeostasis, feedback and motivation. In F. M. Toates \& T. R. Halliday (Eds.), Analysis of motivational processes (pp. 23-37). London: Academic Press.

DREWNOWSKI, A. (1991). Obesity and eating disorders: Cognitive aspects of food preference and food aversion. Bulletin of the Psychonomic Society, 29, 261-264.

Epstein, A. N., Nicolaidis, S., \& Miselis, R. R. (1975). The glucoprivic control of food intake and the glucostatic theory of feeding behaviour. In G. J. Mogenson \& F. R. Calaresu (Eds.), Neural integration of physiological mechanisms and behaviour (pp. 148-168). Toronto: Toronto University Press.

FERNSTROM, J. D. (1983). Role of precursor availability in control of monoamine biosynthesis in brain. Physiological Reviews, 63, 484-546.

Friedman, M. I., \& STricker, E. M. (1976). The physiological psychology of hunger: A physiological perspective. Psychological Review, 83, 409-431.

Holmes, L. J., Smythe, G. A., \& Storlien, L. H. (1989). Monoaminergic activity at the level of the hypothalamus and striatum: Relationship to anticipated feeding and pancreatic insulin responses. Brain Research, 496, 204-210.

Houk, J. C. (1988). Control strategies in physiological systems. FASEB Journal, 2, 97-107.

KASSER, T. R., HaRris, R. B. S., \& MARTIN, R. J. (1985). Level of satiety: Fatty acid and glucose metabolism in three brain sites associated with feeding. American Journal of Physiology, 248, R447-R452.

LEIBOWITZ, S. F. (1988). Hypothalamic paraventricular nucleus: Interaction between $\mathrm{a}_{2}$-noradrenergic system and circulating hormones and nutrients in relation to energy balance. Neuroscience and Biobehavioral Reviews, 12, 101-109.

MorLey, J. E. (1987). Neuropeptide regulation of appetite and weight. Endocrine Reviews, 8, 256-287.

Nicolaidis, S., \& EVEN, P. (1989). Metabolic rate and feeding behavior. Annals of the New York Academy of Sciences, 575, 86-104.

Nicolaidis, S., \& Rowland, N. (1976). Metering of intravenous vs. oral nutrients and the regulation of energy balance. American Journal of Physiology, 231, 661-668.

Nicolaidis, S., \& RowLAND, N. (1977). Intravenous self-feeding: Long term regulation of energy balance in rats. Science, 195, 589-591.

RITTER, S., \& TAYLOR, J. S. (1989). Capsaicin abolishes lipoprivic but not glucoprivic feeding in rats. American Journal of Physiology, 256, R1232-R1239.

RowlAND, N. E. (1988). Peripheral and central satiety factors in neuropeptide Y-induced feeding in rats. Peptides, 9, 989-992.

RowLAND, N. E. (1990). [Studies with glucoprivic agents]. Unpublished raw data.

Rowland, N. E. (1990). On the waterfront: Predictive and reactive regulatory descriptions of thirst and sodium appetite. Physiology \& Behavior, 48, 899-903.

Rowland, N. E., Bellush, L. L., \& Carlton, J. (1985). Metabolic and neurochemical correlates of glucoprivic feeding. Brain Research Bulletin, 14, 617-624.

Rowland, N. E., \& Carlton, J. (1988). Dexfenfluramine: Effects on food intake in various animal models. Clinical Neuropharmacology, 11(Suppl. 1), \$33-S50.

Rowland, N. E., \& STricker, E. M. (1979). Differential effects of glucose and fructose infusions on insulin-induced feeding in rats. Physiology \& Behavior, 22, 387-389.

SCHNEIDER, J., \& WADE, G. (1989). Availability of metabolic fuels controls estrous cyclicity of Syrian hamsters. Science, 244, 1326-1328.

Schwartz, D. H., McClane, S., Hernandez, L., \& Hoebel, B. G. (1989). Feeding increases extracellular serotonin in the lateral hypothalamus of the rat as measured by microdialysis. Brain Research, 479, 349-354.

SCLAFANI, A. (1989). Dietary-induced overeating. Annals of the New York Academy of Sciences, 575, 281-289.

SClafani, A. (1991). Conditioned food preferences. Bulletin of the Psychonomic Society, 29, 256-260. 
Scotr, T. R. (1990). In E. D. Capaldi \& T. L. Powley (Eds.), Taste, experience and feeding (pp. 45-61). Washington, DC: American Psychological Association.

Smith, G. P. (1989). Animal models of human eating disorders. Annals of the New York Academy of Sciences, 575, 63-72.

Souquer, A.-M., \& Rowland, N. E. (1989). Effect of chronic administration of dexfenfluramine on stress- and palatability-induced food intake in rats. Physiology \& Behavior, 46, 145-149.

Stanley, B. G., Anderson, K. C., Grayson, M. H., \& Leibowtrz, S. F. (1989). Repeated hypothalamic stimulation with neuropeptide $Y$ increases daily carbohydrate and fat intake and body weight gain in female rats. Physiology \& Behavior, 46, 173-177.

Stricker, E. M., Rowland, N. E., Saller, C. F., \& Friedman, M. I. (1977). Homeostasis during hypoglycemia: Central control of adrenal secretion and peripheral control of feeding. Science, 196, 79-81.

TORDOFF, M. G., TePper, B. J., \& Friedman, M. I. (1987). Food flavor preferences produced by drinking glucose and oil in normal and diabetic rats: Evidence for conditioning based on fuel oxidation. Physiology \& Behavior, 41, 481-487.

WeINGARTEN, H. (1990). Learning, homeostasis, and the control of feeding behavior. In E. D. Capaldi \& T. L. Powley (Eds.), Taste, experience and feeding (pp. 14-27). Washington, DC: American Psychological Association.

Woods, S. C., \& Kulkosky, P. J. (1976). Classically-conditioned changes of blood glucose level. Psychosomatic Medicine, 38, 201-219.

Woods, S. C., TABorsky, G. J., \& Porte, D. (1986). Central nervous system control of nutrient homeostasis. In F. E. Bloom (Ed.), Handbook of physiology: Sect. 1. The nervous system. Vol. 4. Intrinsic regulatory systems of the brain (pp. 365-411). Bethesda, MD: American Physiological Society.

WURTMAN, R. J., \& WURTMAn, J. J. (1988). Do carbohydrates affect food intake via neurotransmitter activity? Appetite, 11(Suppl.), 42-47. 